\title{
The Effect of Gluten-Free Diet on Thyroid Autoimmunity in Drug-Naïve Women with Hashimoto's Thyroiditis: A Pilot Study
}

\author{
Authors \\ Robert Krysiak, Witold Szkróbka, Bogusław Okopień
}

\begin{abstract}
Affiliation
Department of Internal Medicine and Clinical Pharmacology, Medical University of Silesia, Katowice, Poland
\end{abstract}

Key words

celiac disease, hypothalamic-pituitary-thyroid axis, thyroid function tests, thyroiditis

$\begin{array}{ll}\text { received } & 05.06 .2018 \\ \text { revised } & 26.06 .2018 \\ \text { accepted } & 04.07 .2018\end{array}$

Bibliography

DOI https://doi.org/10.1055/a-0653-7108

Published online: 30.7.2018

Exp Clin Endocrinol Diabetes 2019; 127: 417-422

(c) J. A. Barth Verlag in Georg Thieme Verlag KG Stuttgart . New York

ISSN 0947-7349

\section{Correspondence}

Robert Krysiak

Department of Internal Medicine and Clinical Pharmacology Medical University of Silesia

Medyków 18

40-752 Katowice

Poland

Tel.: + 48/322/523 902, Fax: + 48/322/523902

r.krysiak@interia.pl

\section{ABSTRACT}

Background Autoimmune thyroid disease is often accompanied by celiac disease.
Objective The purpose of this study was to investigate whether a gluten-free diet affects thyroid autoimmunity, hypothalamic-pituitary-thyroid axis activity and thyroid function tests in women with Hashimoto's thyroiditis and incidentally found positive anti-tissue transglutaminase antibodies.

Methods The study included 34 women with autoimmune thyroiditis divided into two group. The patients belonging to the first one (group $A, n=16$ ) complied with the gluten-free diet for 6 months, while the remaining patients (group B, $n=18$ ) remained without any dietary treatment. Serum titers of thyroid peroxidase and thyroglobulin antibodies, as well as serum levels of thyrotropin, free thyroid hormones and 25-hydroxyvitamin D were measured at the beginning of the study and 6 months later. Based on thyrotropin and free thyroid hormone levels, Jostel's thyrotropin index, the SPINA-GT index and the SPINA-GD index were calculated.

Results All patients completed the study protocol. In group $B$, serum thyrotropin and free thyroid hormones levels, serum 25-hydroxyvitamin D levels as well as the calculated indices remained at the similar levels. The gluten-free diet reduced thyroid antibody titers, as well as slightly increased 25-hydroxyvitamin D levels and the SPINA-GT index. In group A, the impact on TPOAb and TgAb titers correlated with the changes in the SPINA-GT index, whereas the impact on TPOAb with the changes in 25-hydroxyvitamin D levels.

Conclusions The obtained results suggest that the glutenfree diet may bring clinical benefits to women with autoimmune thyroid disease.

$\begin{array}{ll}\text { List of abbreviations } \\ \mathrm{CI} & \text { confidence interval } \\ \text { IU } & \text { international unit } \\ \text { SD } & \text { standard deviation } \\ \text { SPINA } & \text { structure parameter inference approach } \\ \text { TgAb } & \text { thyroglobulin antibodies } \\ \text { TPOAb } & \text { thyroid peroxidase antibodies }\end{array}$

\section{Introduction}

Hashimoto's thyroiditis, an autoimmune disorder destroying thyroid follicular cells by cell and antibody-mediated immune processes, is the most common thyroid disease in iodine-sufficient areas and one of the most common human disorders [1,2]. The disease is caused by replacement of follicular cells by lymphocytic infiltrate and fibrosis, and is characterized by the presence of thyroid antibodies, particularly of thyroid peroxidase antibodies (TPOAb) and thyroglobulin antibodies (TgAb) [3,4]. 
The results of many studies evidenced an association between Hashimoto's thyroiditis and coeliac disease or an asymptomatic increase in anti-tissue transglutaminase antibodies [5-8]. Autoimmune thyroiditis is the most prevalent coexisting autoimmune disorder in patients with celiac disease [9]. A pooled analysis, including 6024 patients with autoimmune thyroiditis, found a markedly increased prevalence of biopsy-confirmed coeliac disease, allowing the authors to conclude that all patients with autoimmune thyroiditis should be screened for the presence of coeliac disease [10]. The association between autoimmune thyroid disease and coeliac disease may be explained by low selenium [11] or vitamin D [12] status secondary to their malabsorption, the interaction of tissue transglutaminase-2 IgA antibodies to thyroid follicles and thyroid extracellular matrix [13], or by a shared immunogenetic make-up [14]. Both exogenous vitamin D (cholecalciferol) [15] and selenium [16] seem to play a role in the development and progression of Hashimoto's thyroiditis.

To best of our knowledge, no previous study investigated whether restriction of gluten intake affect thyroid autoimmunity and function. Therefore, the purpose of our study was to investigate whether a gluten-free diet has an impact on thyroid autoimmunity, hypothalamic-pituitary-thyroid axis activity and thyroid function tests in drug-naïve women with Hashimoto's thyroiditis.

\section{Materials and Methods}

\section{Patients}

The participants of the study were selected among young women, aged between 20 and 45 years, with recently diagnosed and previously untreated autoimmune thyroiditis. To be admitted to the study, they were required to have (a) positive TPOAb (>100U/mL), (b) the reduced echogenicity of the thyroid parenchyma on thyroid ultrasonography; (c) normal thyroid function (thyrotropin levels in the range between 0.4 and $4.5 \mathrm{mU} / \mathrm{L}$, free thyroxine in the range between 10.0 and $21.0 \mathrm{pmol} / \mathrm{L}$ and free triiodothyronine in the range between 2.6 and $6.5 \mathrm{pmol} / \mathrm{L}$ ) and (d) incidentally found positive anti-tissue transglutaminase antibodies without clinical symptoms of coeliac disease. All patients ingested gluten in their diet prior to starting the study.

We excluded women with symptomatic coeliac disease, positive antibodies against thyrotropin receptor, diabetes or other endocrine disorders, impaired renal or hepatic function, any acute and chronic inflammatory processes, any other serious disorders, pregnancy or lactation, as well as women receiving any chronic treatment.

The research was conducted in accordance with the Helsinki Declaration. The study was approved by the local review board and written, informed consent was obtained from each participant.

\section{Study design}

Before the study, all participants were informed about the benefits and risks of following a gluten-free diet. Based on patient preference, the participants were allocated to one of two groups. Patients belonging to group $A(n=16)$ were instructed by a physician and a professional dietitian with the help of leaflets to follow a glutenfree diet. Patients from group B $(n=18)$ were not prescribed any special dietary recommendations. Participants were seen every two months to ensure adherence to the diet and to boost compliance with the protocol. During each visit, women were asked to complete a questionnaire evaluating how often in the past two months they had consumed each of the twenty most commonly used meals of Polish cuisine. Food intake frequency was assessed in terms of the following six categories: everyday, 5-6 times per week, 3-4 times per week, 1-2 times per week, less than once per week and never. The questionnaire included also questions on the use of gluten-free products. Moreover, each patient was required to provide the packaging and labels of the gluten-free products he or she consumed.

\section{Laboratory assays}

Laboratory assays were performed in duplicate (to minimize analytical errors) at baseline and 6 month later (at the end of the treatment period). Venous blood samples were collected from the antecubital vein between 8 and 9 a.m. (to avoid possible circadian fluctuations in the parameters studied) after an overnight 12-h fasting. Serum levels of thyrotropin, free thyroxine and free triiodothyronine and titers of TPOAb and TgAb were measured by direct chemiluminescence using acridinium ester technology (ADVIA Centaur XP Immunoassay System, Siemens Healthcare Diagnostics, Munich, Germany). Serum 25-hydroxyvitamin D levels were assayed by competitive immunoassay using Roche Diagnostic commercial kits and a multichannel automatic analyzer (Roche Cobas e 411, Mannheim, Germany). Immunoglobulins A against the tissue transglutaminase antigen were detected using the enzymelinked immunosorbent assay method (Euroimmun, Lübeck, Germany). Jostel's thyrotropin index, the structure parameter inference approach (SPINA)-GT index as well as the SPINA-GD indices were calculated by the investigators based on thyrotropin and free thyroid hormone levels using SPINA-Thyr 4.0.1 for Windows software in accordance with the formulas described previously [17-19].

\section{Statistical analysis}

Values for hormones, antibodies and thyroid function tests were natural log-transformed to yield a normal distribution for statistical analyses. Treatment groups were compared using the $t$ test for independent samples. The differences between the means of variables within the same treatment group were analyzed with Student's paired t test. The clinical importance of the result was assessed based on the $95 \%$ confidence interval. A t statistic and two sample means were used to generate an interval estimate of the difference between two population means. Categorical variables were analyzed by $x^{2}$ test, while Pearson's r-tests were used to calculate the significance of each correlation. Differences were described as statistically significant if $95 \%$ confidence intervals did not include the null value and/or two-tailed p values were below 0.05 .

\section{Results}

At study entry, both groups of patients were comparable with respect to age, body mass index, smoking, the percentage of women giving birth in the past, the number of deliveries, as well as serum levels of hormones and 25-hydroxyvitamin D, serum titers of thyroid antibodies, Jostel's thyrotropin index, the SPINA-GT index and 
- Table 1 Baseline characteristics of patients.

\begin{tabular}{|l|l|l|l|}
\hline Variable & \multicolumn{1}{|c|}{ Gluten-free diet (Group A) } & Gluten-containing diet (Group B) & Difference [95\% CI] \\
\hline Number of patients [n] & 16 & 18 & - \\
\hline Age [years; mean (SD)] & $30(5)$ & $31(6)$ & $1[-3,5]$ \\
\hline Smokers [\%] & 25 & 22 & - \\
\hline Body mass index [kg/m²; mean (SD)] & $22.9(2.3)$ & $67 / 1.2(0.9)$ & $0.2[-1.3,1.7]$ \\
\hline $\begin{array}{l}\text { Past deliveries (\%)/Number of } \\
\text { deliveries [n; mean (SD)] }\end{array}$ & $69 / 1.1(0.9)$ & & $0.1[-0.5,0.7]$ \\
\hline
\end{tabular}

Cl: confidence interval; IU: international unit; SD: standard deviation; SPINA: structure parameter inference approach; TgAb: thyroglobulin antibodies;

TPOAb: thyroid peroxidase antibodies

the SPINA-GD index ( $\triangleright$ Table 1, 2). The gluten-free diet was well tolerated and no patient prematurely terminated the study. With the exception of gluten-free and gluten-containing products, the study groups did not differ in the frequency of particular food consumption. During the follow-up period, no patient developed clinical symptoms of coeliac disease. At the end of the study, positive anti-tissue transglutaminase antibodies were present in sera of 6 patients (38\%) from group A and in all 18 patients from group $B$.

The gluten-free diet reduced serum titers of TPOAb and TgAb, as well as increased serum levels of 25-hydroxyvitamin $D$ and the SPINA-GT index ( $\triangleright$ Table 2). The gluten-free diet did not affect thyrotropin, free thyroid hormones, Jostel's thyrotropin index and the SPINA-GD index. In group B, serum thyroid antigens, thyrotropin, free thyroxine, free triiodothyronine and 25-hydroxyvitamin $D$ as well as Jostel's thyrotropin, the SPINA-GD and the SPINA-GT indices remained at the similar levels throughout the study. At the end of the study, there were significant differences between both groups in thyroid antibody titers, 25-hydroxyvitamin D levels and the SPINA-GT index ( $\triangleright$ Table 2 ).

At entry, TPOAb titers correlated with TgAb titers $(r=0.60$, $p<0.001)$. Antibody titers inversely correlated with 25 -hydroxyvitamin D levels (TPOAb: $r=-0.37, p<0.01$; TgAb: $r=-0.25$, $p<0.05)$. Titers of TPOAb and TgAb correlated also with the SPINAGT index (TPOAb: $r=-0.35, p<0.01$;gAb: $r=-0.29, p<0.05)$. In group A, the impact of treatment on TPOAb titers correlated with the changes in TgAb titers $(r=0.52, p<0.001)$ and with the changes in 25-hydroxyvitamin D levels $(r=0.34, p<0.05)$. The gluten-free diet-induced changes in antibody titers correlated also with the impact on the SPINA-GT index (TPOAb: $r=0.40, p<0.001$; TgAb: $r=0.32, p<0.05)$. No other correlations were found.

\section{Discussion}

The major finding of our study is that the gluten-free diet reduced thyroid autoimmunity and slightly increased thyroid output in euthyroid women with Hashimoto's thyroiditis. This action seems to be a specific effect of the gluten-free diet because in a significant proportion of patients (62\%) the decrease in thyroid antibody titers and changes in thyroid function tests were accompanied by disappearance of anti-tissue transglutaminase antibodies. The obtained results cannot be explained by seasonal fluctuations or timedependent changes in antibody titers and thyroid function tests, because serum levels of thyrotropin and free thyroid hormones, serum titers of TPOAb and $\mathrm{TgAb}$, as well as Jostel's thyrotropin, the SPINA-GT and SPINA-GD indices in individuals not limiting gluten intake remained at the similar levels during the whole study period. Considering very strict inclusion and exclusion criteria, the consequence of which was obtaining a homogenous group of patients with autoimmune thyroiditis, the results of our study cannot be attributed to coexisting disorders or to the action of any drugs taken by patients. Finally, the obtained results do not seem to be a consequence of the improvement in the diet composition because analysis of individual food-frequency questionnaires revealed no significant differences in particular food consumption, including fish or milk intake.

It is difficult to explain mechanisms responsible for the favorable effect of the gluten-free diet on thyroid autoimmunity. Our research indicates that one of them is an improvement in vitamin $D$ status. In line with this hypothesis, our population of patients was characterized by relatively low serum levels of 25-hydroxyvitamin $D$, being is the best marker determining vitamin D status [20]. Moreover, the gluten-free diet, but not a normal diet, increased circulating levels of 25-hydroxyvitamin $D$, and this action correlated with the changes in TPOAb titers. Interestingly, exogenous vitamin $D$ preparations reduced thyroid antibody titers in women with thyroid autoimmunity, and this effect was stronger for TPOAb than for TgAb [21], which probably reflects the fact that TPOAb are characterized by higher sensitivity and at least equal specificity to $\mathrm{TgAb}$ in the diagnosis of autoimmune thyroid disease [22]. Taking into account that correlations between TPOAb titers and 25-hydroxyvitamin D levels were moderate, it seems that also other mechanisms contribute to the favorable effect of the gluten-free diet. One of them may be an improvement in selenium status, because preparations of this micronutrient were found to reduce thyroid autoimmunity, at least in part by inhibiting a secretory function of human T cells [16]. Apparently healthy women inhabiting the Upper Silesia, where the study was conducted, are characterized by low selenium status [23], which might have been even worse in the participants of our study. Because no small intestinal biopsy was performed, it is possible that a significant proportion of our patients might have had subclinical (asymptomatic) coeliac disease. Coeliac disease, even mild, is characterized by selenium depletion [24], more pronounced in subjects poorly complying with the gluten-free diet than in well-complying patients [25]. We did not measure serum or whole-blood selenium levels because in the adult population they do not correlate well with dietary consumption of this micronutrient, reflecting better intake of selenomethionine and selenium-enriched yeast supplements than intake of the remaining forms of selenium [26]. However, relatively low baseline values of the SPINA-GD index in both groups of patients indirectly 
- Table 2 The effect of the gluten-free diet on thyroid antibody titers, hormones, thyroid function tests and 25-hydroxyvitamin D levels in euthyroid women with Hashimoto's thyroiditis.

\begin{tabular}{|c|c|c|c|}
\hline Variable & Gluten-free diet (Group A) & Gluten-containing diet (Group B) & Difference $[95 \% \mathrm{Cl}]$ \\
\hline \multicolumn{4}{|c|}{ TPOAb [U/mL; mean (SD)] } \\
\hline Baseline & $925(265)$ & $891(242)$ & $-34[-211,143]$ \\
\hline After 6 months & $705(206)^{\#}$ & $920(280)$ & $215[42,388]^{*}$ \\
\hline Change & $-200(105)$ & $29(25)$ & $229[177,281]^{\&}$ \\
\hline \multicolumn{4}{|c|}{$\operatorname{TgAb}[\mathrm{U} / \mathrm{mL} ;$ mean (SD)] } \\
\hline Baseline & $832(311)$ & $792(274)$ & $-40[-244,164]$ \\
\hline After 6 months & $629(240)^{\#}$ & $845(324)$ & $216[15,417]^{*}$ \\
\hline Change & $-203(120)$ & $53(58)$ & $256[194,318]^{\&}$ \\
\hline \multicolumn{4}{|c|}{ Thyrotropin [mIU/L; mean (SD)] } \\
\hline Baseline & $2.7(1.0)$ & $2.9(0.8)$ & $0.2[-0.4,0.8]$ \\
\hline After 6 months & $2.4(0.8)$ & $2.6(0.9)$ & $0.2[-0.4,0.8]$ \\
\hline Change & $-0.3(0.2)$ & $-0.3(0.2)$ & $0.0[-0.1,0.1]$ \\
\hline \multicolumn{4}{|c|}{ Free thyroxine [pmol/L; mean (SD)] } \\
\hline Baseline & $14.9(2.3)$ & $15.3(2.7)$ & $0.4[-1.4,2.2]$ \\
\hline After 6 months & $16.1(2.4)$ & $15.0(2.3)$ & $-1.1[-2.7,0.5]$ \\
\hline Change & $1.2(1.4)$ & $0.3(0.6)$ & $-0.9[-1.9,0.1]$ \\
\hline \multicolumn{4}{|c|}{ Free triiodothyronine [pmol/L; mean (SD)] } \\
\hline Baseline & $3.2(0.6)$ & $3.1(0.6)$ & $-0.1[-0.5,0.3]$ \\
\hline After 6 months & $3.6(0.7)$ & $3.2(0.7)$ & $-0.4[-0.9,0.1]$ \\
\hline Change & $0.4(0.3)$ & $0.1(0.3)$ & $-0.3[-0.1,0.5]$ \\
\hline \multicolumn{4}{|c|}{ Jostel's thyrotropin index [mean (SD)] ${ }^{1}$} \\
\hline Baseline & $3.0(0.2)$ & $3.1(0.3)$ & $0.1[-0.1,0.3]$ \\
\hline After 6 months & $3.0(0.2)$ & $3.0(0.2)$ & $0.0[-0.1,0.1]$ \\
\hline Change & $0.0(0.1)$ & $-0.1(0.2)$ & $-0.1[-0.3,0.1]$ \\
\hline \multicolumn{4}{|c|}{ SPINA-GT index $[\mathrm{pmol} / \mathrm{s} \text {; mean (SD) }]^{2}$} \\
\hline Baseline & $2.28(0.38)$ & $2.26(0.34)$ & $-0.02[-0.27,0.23]$ \\
\hline After 6 months & $2.62(0.40)^{\#}$ & $2.30(0.37)$ & $-0.32[-0.59,-0.05]^{*}$ \\
\hline Change & $0.34(0.12)$ & $0.04(0.06)$ & $-0.3[-0.37,-0.23]^{\&}$ \\
\hline \multicolumn{4}{|c|}{ SPINA-GD index $[\mathrm{nmol} / \mathrm{s} ; \text { mean }(\mathrm{SD})]^{3}$} \\
\hline Baseline & $19.86(3.04)$ & $18.73(2.53)$ & $-1.13[-3.08,0.82]$ \\
\hline After 6 months & $20.68(2.41)$ & $19.73(2.81)$ & $-0.95[-2.79,0.89]$ \\
\hline Change & $0.82(0.25)$ & $1.00(0.31)$ & $0.18[-0.02,0.38]$ \\
\hline \multicolumn{4}{|c|}{ 25-hydroxyvitamin $\mathrm{D}$ [ng/mL; mean (SD)] } \\
\hline Baseline & $20(6)$ & $21(5)$ & $1[-3,5]$ \\
\hline After 6 months & $25(6)^{\#}$ & $20(5)$ & $-5[-9,-1]^{*}$ \\
\hline Change & $5(3)$ & $-1(2)$ & $-6[-8,-4]^{\&}$ \\
\hline \multicolumn{4}{|c|}{$\begin{array}{l}\text { Cl: confidence interval; IU: international unit; SD: standard deviation; SPINA: structure parameter inference approach; TgAb: thyroglobulin antibodies; } \\
\text { TPOAb: thyroid peroxidase antibodies; }{ }^{1} \text { Reference range: } 1.3-4.1 \text { [17]; }{ }^{2} \text { Reference range: } 1.4-8.7 \text { pmol/s [18]; }{ }^{3} \text { Reference range: } 20-60 \mathrm{nmol} / \mathrm{s} \\
\text { [18]; * statistically significant difference between both groups; }{ }^{*} \text { statistically significant difference between post-treatment and baseline values in the } \\
\text { same group; }{ }^{\&} \text { statistically significant difference between the changes in both treatment groups. }\end{array}$} \\
\hline
\end{tabular}

support selenium deficiency in the study population. The glutenfree diet may also directly inhibit activity of inflammatory cells. In line with this explanation, dietary gluten was found to alter the balance of pro-inflammatory and anti-inflammatory cytokines in $\mathrm{T}$ cells of mice towards a more inflammatory cytokine profile [27], while the gluten-free diet reduced circulating levels of proinflammatory cytokines [28].

The gluten-free diet induced an increase in the SPINA-GT index, being a marker of the maximum secretion rate under stimulated conditions $[18,19]$. Its retest reliability is higher than that of thy- rotropin, free thyroxine and free triiodothyronine [18], and this fact may explain why, despite affecting the SPINA-GT index, the dietary treatment had a neutral effect on serum hormone levels. The impact on thyroid output was relatively small, because during the whole period of the follow-up values of the SPINA-GT index remained within the reference range. Interestingly, treatment-induced changes in the SPINA-GT correlated with both baseline serum titers of TPOAb and TgAb and with the reduction in thyroid antibody titers. This finding allows us to draw two conclusions. Firstly, the inhibitory effect of the gluten-free diet on thyroid auto- 
immunity may participate in the improvement of thyroid secretory capacity. Secondly, the effect on antibody titers was strongest in patients with the highest antibody titers. Considering a predictive value of thyroid antibodies and their titers as risk factors of the development of hypothyroidism [29, 30], the gluten-free diet may delay the development of thyroid hypofunction in euthyroid women with Hashimoto's thyroiditis. Despite increasing the SPINA-GT index, the gluten-free diet exerted no effect of the other calculated parameters of thyroid homeostasis. A neutral effect on the SPINA-GD index, estimating the sum of activity of peripheral deiodinases [19], means that the gluten-free diet does not affect peripheral metabolism of thyroid hormones. Finally, no change in Jostel's thyrotropin index, a quantitative marker for pituitary thyrotropic function [17], indicates that the gluten-free diet is devoid of a direct effect on thyrotropic cells.

Several shortcomings of our study bear mentioning. The most important limitation of the study is its non-randomized nature, a small number of participants and a short period of therapy. Moreover, the study population was characterized by low selenium status [23] and adequate iodine intake [31]. It is not certain that the impact of the gluten-free diet is similar in selenium-sufficient and/ or iodine-depleted areas. Furthermore, because the study included only euthyroid women, the question whether the gluten-free diet affects antibody titers and thyroid function tests in subjects with thyroid hypofunction remains unanswered. Finally, it cannot be ruled out that the effect of the gluten-free diet may be different in women treated with agents reducing thyroid antibody titers (levothyroxine, vitamin D and selenium), who were not included in our study.

Summing up, the gluten-free diet reduced serum titers of TPOAb and TGAb in euthyroid women with Hashimoto's thyroiditis, which correlated with the increase in the SPINA-GT index. This finding indicates that the gluten free-diet may bring clinical benefits to euthyroid women with Hashimoto's thyroiditis, who, because of markedly elevated thyroid antibody titers, are at high risk of the development of hypothyroidism. Because of study limitations, our study should be regarded as a pilot one, and larger prospective trials are required to support our findings.

\section{Acknowledgements}

The study was supported by the statutory grant of the Medical University of Silesia (KNW-1-062/N/7/0). None of the authors has any conflict of interest. The experiments comply with the current law of Poland.

\section{Funding}

The study was supported by the statutory grant of the Medical University of Silesia (KNW-1-062/N/7/0). The experiments comply with the current law of Poland.

\section{Conflict of Interest}

The authors declare that they have no conflict of interest.
Institutional approval: The study was approved by the Bioethical Committee of the Medical University of Silesia.

\section{References}

[1] Lorini R, Gastaldi R, Traggiai C et al. Hashimoto's thyroiditis. Pediatr Endocrinol Rev 2003; 1: (Suppl 2): 205-211

[2] Caturegli P, Kimura H, Rocchi R et al. Autoimmune thyroid diseases. Curr Opin Rheumatol 2007; 19: 44-48

[3] Hiromatsu Y, Satoh H, Amino N. Hashimoto's thyroiditis: History and future outlook. Hormones (Athens) 2013; 12: 12-18

[4] Brown RS. Autoimmune thyroid disease: Unlocking a complex puzzle. Curr Opin Pediatr 2009; 21: 523-528

[5] Tuhan H, Işık S, Abacı A et al. Celiac disease in children and adolescents with Hashimoto thyroiditis. Turk Pediatri Ars 2016; 51: 100-105

[6] Hadithi M, de Boer H, Meijer JW et al Coeliac disease in Dutch patients with Hashimoto's thyroiditis and vice versa. World J Gastroenterol 2007; 13: 1715-1722

[7] Ruggeri RM, Trimarchi F, Giuffrida G et al. Autoimmune comorbidities in Hashimoto's thyroiditis: Different patterns of association in adulthood and childhood/adolescence. Eur J Endocrinol 2017; 176: 133-141

[8] Fallahi P, Ferrari SM, Ruffilli I et al. The association of other autoimmune diseases in patients with autoimmune thyroiditis: Review of the literature and report of a large series of patients. Autoimmun Rev 2016; 15: 1125-1128

[9] Demirezer Bolat A, Akin FE, Tahtaci M et al. Risk factors for polyautoimmunity among patients with celiac disease: A cross-sectional survey. Digestion 2015; 92: 185-189

[10] Roy A, Laszkowska M, Sundström J et al. Prevalence of celiac disease in patients with autoimmune thyroid disease: A meta-analysis. Thyroid 2016; 26: 880-890

[11] Stazi AV, Trinti B. Selenium status and over-expression of interleukin-15 in celiac disease and autoimmune thyroid disease. Ann Ist Super Sanita 2010; 46: 389-399

[12] Fisher AH, Lomasky SJ, Fisher MJ et al. Celiac disease and the endocrinologist: A diagnostic opportunity. Endocr Pract 2008; 14: 381-388

[13] Naiyer AJ, Shah J, Hernandez L et al. Tissue transglutaminase antibodies in individuals with celiac disease bind to thyroid follicles and extracellular matrix and may contribute to thyroid dysfunction. Thyroid 2008; 18: 1171-1178

[14] Kahaly G], Frommer L. Polyglandular autoimmune syndromes. J Endocrinol Invest 2018; 41: 91-98

[15] Mansournia N, Mansournia MA, Saeedi S et al. The association between serum 25-OHD levels and hypothyroid Hashimoto's thyroiditis. J Endocrinol Invest 2014; 37: 473-476

[16] Krysiak R, Okopień B. The effect of levothyroxine and selenomethionine on lymphocyte and monocyte cytokine release in women with Hashimoto's thyroiditis. J Clin Endocrinol Metab 2011; 96: 2206-2215

[17] Jostel A, Ryder WD, Shalet SM. The use of thyroid function tests in the diagnosis of hypopituitarism: Definition and evaluation of the TSH index. Clin Endocrinol 2009; 71: 529-534

[18] Dietrich JW, Müller P, Schiedat F et al. Nonthyroidal illness syndrome in cardiac illness involves elevated concentrations of 3,5-diiodothyronine and correlates with atrial remodeling. Eur Thyroid J 2015; 4: 129-137

[19] Dietrich JW, Landgrafe-Mende G, Wiora E et al. Calculated parameters of thyroid homeostasis: Emerging tools for differential diagnosis and clinical research. Front Endocrinol (Lausanne) 2016; 7: 57

[20] Lips P. Vitamin D physiology. Prog Biophys Mol Biol 2006; 92: 4-8 
[21] Krysiak R, Kowalcze K, Okopień B. The effect of vitamin D on thyroid autoimmunity in non-lactating women with postpartum thyroiditis. Eur ] Clin Nutr 2016; 70: 637-639

[22] Muller AF, Drexhage HA, Berghout A. Postpartum thyroiditis and autoimmune thyroiditis in women of childbearing age: Recent insights and consequences for antenatal and postnatal care. Endocr Rev 2001; 22: $605-630$

[23] Kłapcińska B, Poprzecki S, Danch A et al. Selenium levels in blood of Upper Silesian population: Evidence of suboptimal selenium status in a significant percentage of the population. Biol Trace Elem Res 2005; 108: $1-15$

[24] Hinks L], Inwards KD, Lloyd B et al. Body content of selenium in coeliac disease. Br Med J (Clin Res Ed) 1984; 288: 1862-1863

[25] Kalita B, Nowak P, Slimok M et al. Selenium plasma concentrations in children with celiac disease in different stages of diagnosis. Pol Merkur Lekarski 2002; 12: 43-44

[26] Hurst R, Collings R, Harvey LJ et al. EURRECA-estimating selenium requirements for deriving dietary reference values. Crit Rev Food Sci Nutr 2013; 53: 1077-1096
[27] Antvorskov JC, Fundova P, Buschard K et al. Dietary gluten alters the balance of pro-inflammatory and anti-inflammatory cytokinws in $\mathrm{T}$ cells of BALB/c mice. Immunology 2013; 13: 23-34

[28] Street ME, Volta C, Ziveri MA et al. Changes and relationships of IGFS and IGFBPS and cytokines in coeliac disease at diagnosis and on gluten-free diet. Clin Endocrinol (Oxf) 2008; 68: 22-28

[29] Chaker L, Bianco AC, Jonklaas J et al. Hypothyroidism. Lancet 2017; 390: $1550-1562$

[30] Arrigo T, Wasniewska M, Crisafulli $G$ et al. Subclinical hypothyroidism: The state of the art. J Endocrinol Invest 2008; 31: 79-84

[31] Szybiński Z.Polish council for control of iodine deficiency disorders. Work of the Polish council for control of iodine deficiency disorders, and the model of iodine prophylaxis in Poland. Endokrynol Polska 2012; 63: 156-160 